\title{
The Concealed Evidence - Case Report
}

\section{Rajiv Dua* \\ Department of Chemistry \& Home (Police), State Forensic Science Laboratory, India}

Received: 海 August 20, 2018; Published: 眥 August 29, 2018

*Corresponding author: Rajiv Dua, Department of Chemistry \& Home (Police), Scientific Officer, State Forensic Science Laboratory, Government of Madhya Pradesh, India

\begin{abstract}
Mobile crime scene investigating team plays an important role to sorting numerous important and sensational crimes. It is important that the crime scene investigator recognize that physical evidence recovered from these scenes may extend well beyond the conveyance itself. The nature of the crime may give the investigator an idea of the type of evidence present. Over the years, criminals have tried many ingenious ways to hide, clean up and remove evidence such as blood, weapons and tools but it's an area where criminal justice technology has always stayed one step ahead of them. In the present paper an interesting case of murder of man was unravelled by the crime scene team. During careful scientific observation of crime scene by the forensic team, it was advised to the Investigating Officer (I.O.) that homicide was done by deceased brother.
\end{abstract}

Keywords: Crime Scene; Homicide; Blood; Incised Wound

\section{Introduction}

Blood is the most common and perhaps most important form of physical trace and fluid evidences in criminal justice today. Its presence always links a suspect and victim to one another and to the scene of the crime [1]. Thorough visual and chemical identification of bloodstains provide crucial information criminal investigation of violent crimes involving blood traces, particularly violent crimes but also other types of criminal events where blood is one of the resulting evidences on the crime scene [2,3]. During a crime event, external forces acting on a body bring about injuries that often result in the expulsion of blood as blood drops or flows. Interactions between the internal forces of surface tension, viscosity and internal cohesion and external forces of gravity, air resistance and applications of force determine the manner in which blood is expelled. These interactions determine with certain predictability, the nature and formation of blood drops, the forces required to disperse blood from a source and how blood behaves upon interaction with external forces [4,5]. Analysis of bloodstains, spatters and the patterns formed by bloodletting events at crime scene it can be determined was it a murder or a suicide, was the injury deliberate or was it the result of helping the victim, what was the sequence and the dynamics of events during the commission of the crime, approximately how long did it take to commit the crime and how much time has elapsed after the formation of those bloodstains, what are subsequent events that took place after the crime was committed, and which was the direction of movement of persons involved after the crime was committed [6,7].

\section{Case History}

A dead body of man was recovered from the pit hole at distance of 5 feet nearby Road (Figure1). The body was already recognized, and forensic team was called to examine the body at crime scene as per forensic requirements.

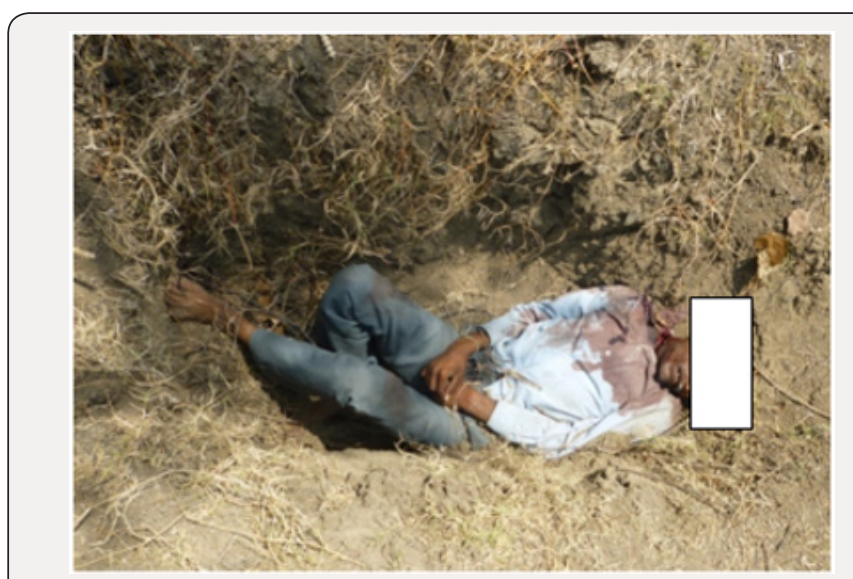

Figure 1: Death body of deceased recovered from pit hole.

\section{Observation and Findings}

At the crime scene, following observation were made 
a. The body was of a man aged about 45 years

b. Shirt from left side of the collar was found to be torn and smudged in blood (Figure 2).

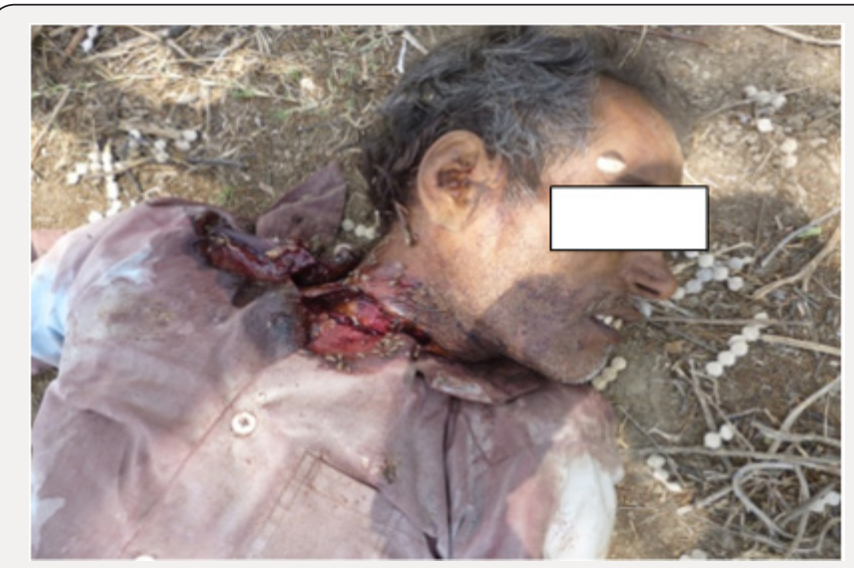

Figure 2: Shirt torn from left side of the collar and smudged with blood.

c. Two plastic shoes were recovered and kept outside the pit hole at a distance of 6 feet from the deceased body

d. One blood stained towel was recovered and kept outside the pit hole at a distance of 5 feet from the deceased body

e. Had a incised wound over the anterior of the neck of dimension $14 \times 7.5 \times 7.5 \mathrm{~cm}$ (Figure 3)

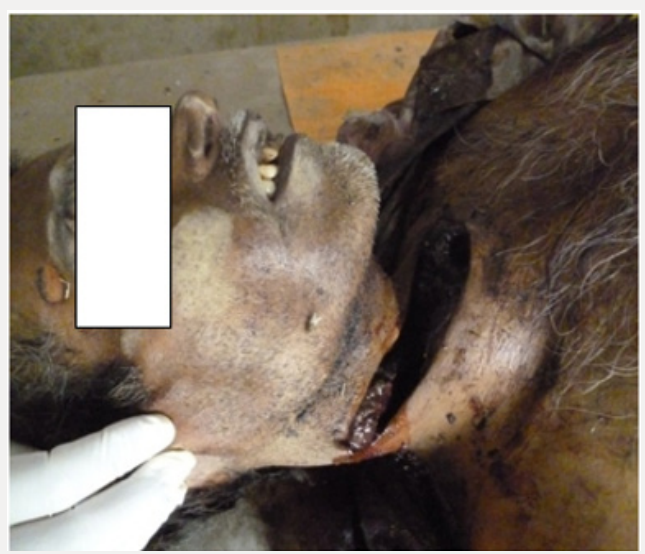

Figure 3: Incised wound over the anterior of the neck of dimension $14 \times 7.5 \times 7.5 \mathrm{~cm}$.

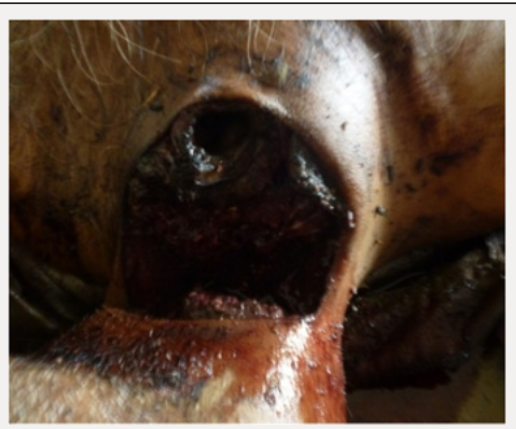

Figure 4: Deep wound showing high impact by hard and sharp object. f. Deep wound showing high impact by hard and sharp object (Figure 4).

Deceased younger brother was sitting adjacent to dead body and was crying. Careful scientific investigation was done by the forensic team and by collecting all supplementary information from concerned village people, it was advised to the investigating Officer (I.O.) that crime scene is secondary and suspected person in this homicidal episode would be deceased younger brother. After establishing the factual position, our second responsibility was to locate primary scene of crime and to gather evidence from there.

\section{Primary Scene of Crime and Evidence Collection}
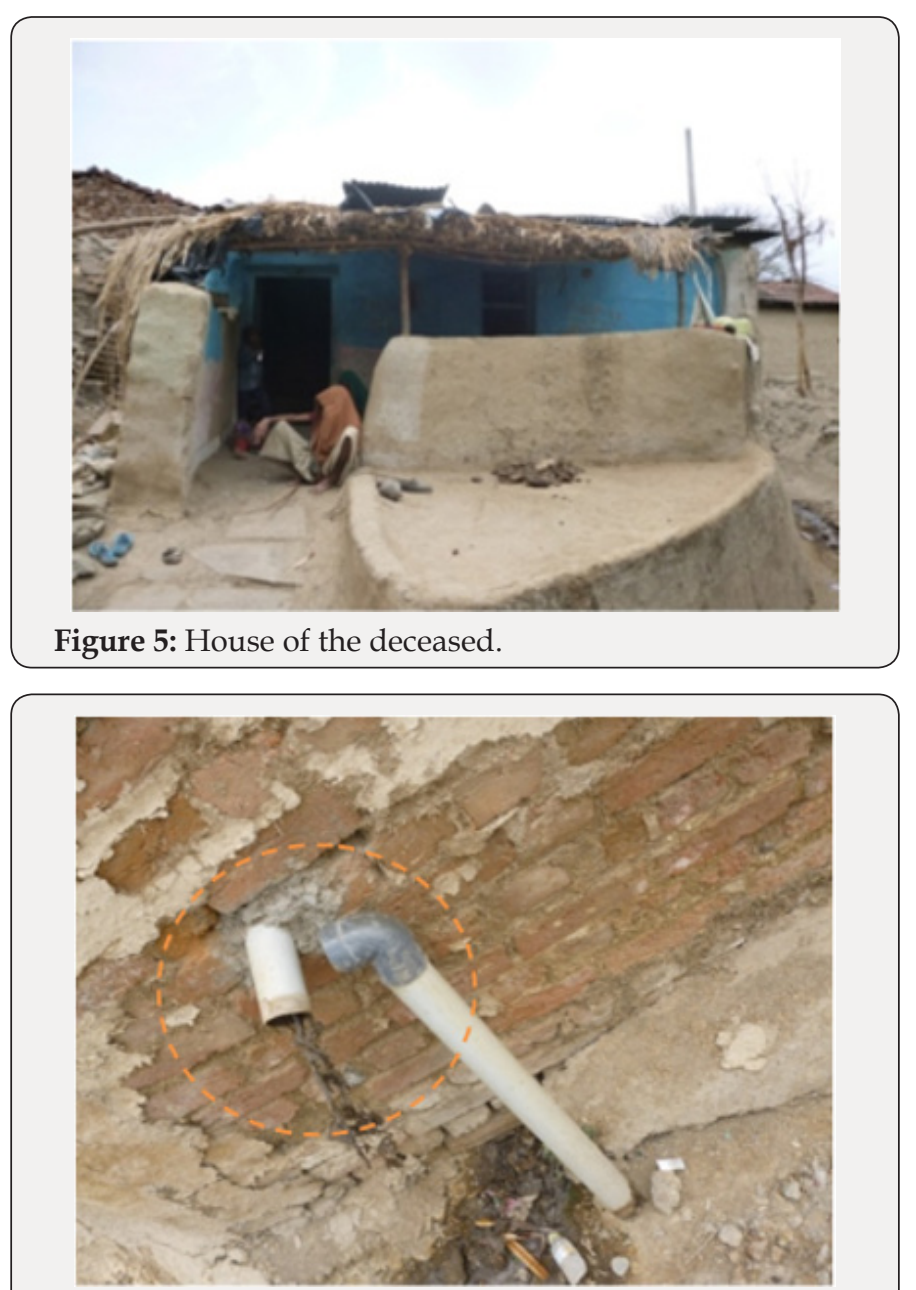

Figure 6: Outlet of P.V.C pipe from the deceased house.

Deceased house was about 500 meters away from the Road. House consist of three room; first room is goat pen (goat shelter) and wash area is there at right side of the room (Figure 5). Second room is living area and kitchen of the deceased and third room is living area of deceased brother where locked wheat storage tank filled completely with wheat and 5 to 6 wheat sack were kept adjacent to western wall of the room. Thorough search of all the room of deceased house was done; no physical evidence was recovered. Forensic and Investigation team was returning back suddenly eye went on washing cloth brush kept at wash area in the 
first room suspected this might be used for cleaning the weapon. Forensic team performed preliminary test of blood (Benzidine test) on the brush which came out to be positive. Then forensic team conducted the preliminary test of blood (Benzidine) on the outlet of wash area i.e. P.V.C pipe from the house of the deceased, it came out be positive (Figures $6 \& 7$ ).

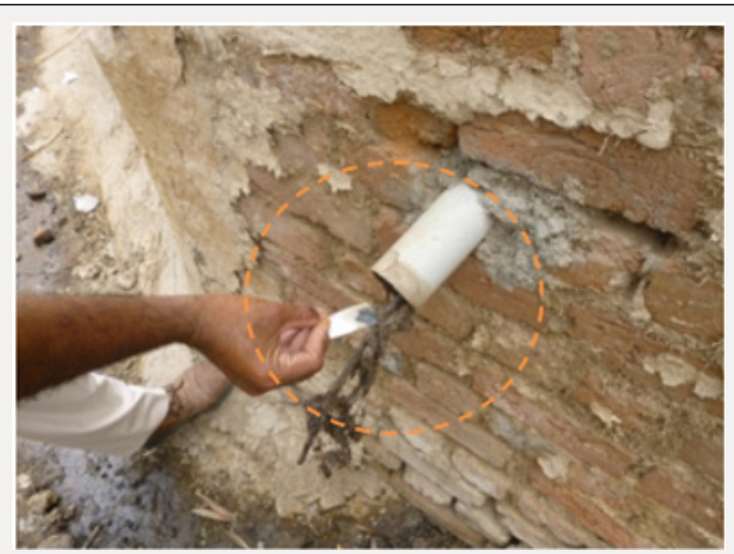

Figure 7: Positive preliminary test of blood on the P.V.C outlet.

On the basis of evidence of presence of blood on clothes washing brush and PVC outlet further confirmed the involvement of someone who belonged to him or someone of relatives may be involved or possibility of any familiar person may be involved. To link the deceased family member with the crime, a thorough investigation of suspected, suspected wife and deceased wife was done and thorough search of deceased house was done again, finally forensic and investigation team got success in searching all the physical evidence from wheat storage container and blood stained weapon (axe) kept inside the wheat sack kept adjacent to the western wall of the room (Figures $8 \& 9$ ). All the questionable blood stains were chemically tested for the presence of blood using a Benzidine test.

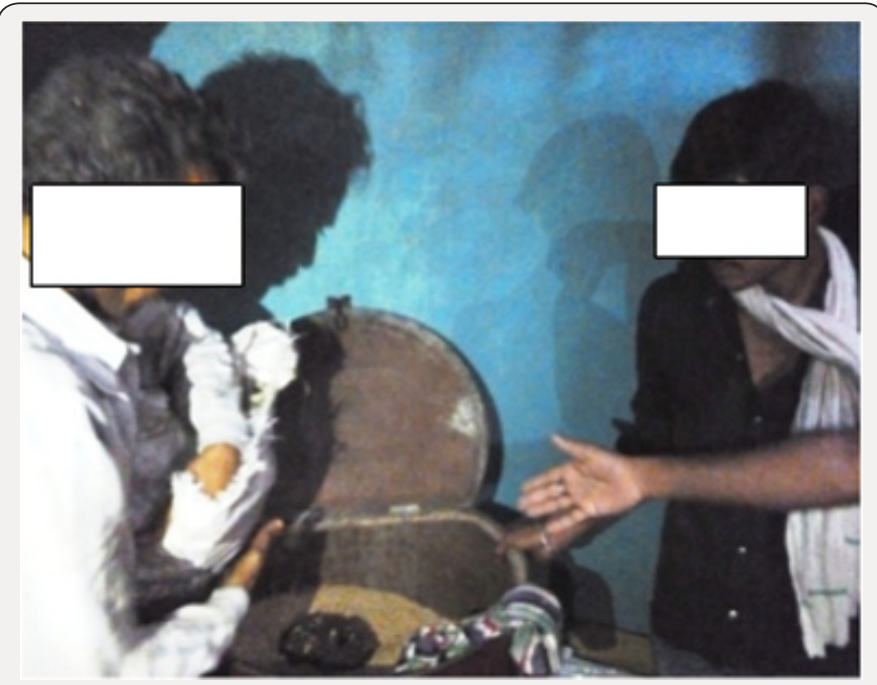

Figure 8: Recovery of physical evidences from the wheat storage container.

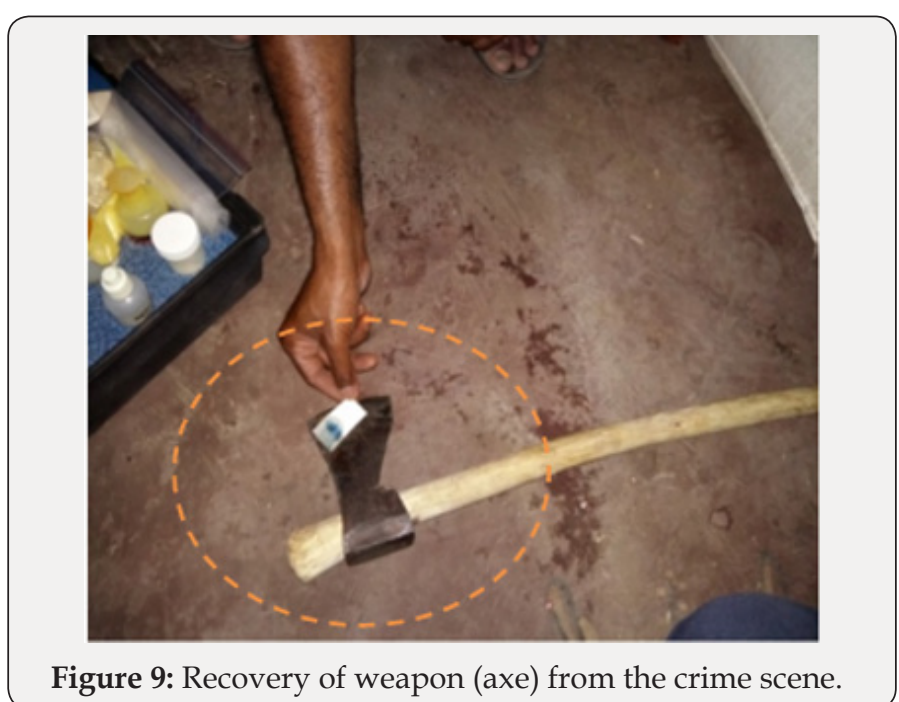

\section{Recovery of physical evidence from wheat storage container}

The following physical evidences were recovered from the plastic bag kept in the wheat container:

a) Red Black Check Shirt of accused smudged with blood.

b) Brown coloured pant of accused stained with blood.

c) Big sized blood smudged golden coloured mattress.

d) Small sized blood smudged red coloured mattress.

e) Blood stained Jute Sack.

f) Blood stained black printed saree.

Blood smeared soil was also recovered from the scene of the crime which was already cleaned by deceased brother. All the samples were subjected to confirmatory blood tests for detection and determination of origin and weapon (axe) was sent for cut mark examination and biological examination.

\section{Laboratory Examination}

The exhibit collected from deceased body after the postmortem, exhibits recovered from the suspect, exhibits seized from the crime scene were sent to forensic science laboratory for confirmatory analysis. Toxicological analysis of deceased viscera came negative and cut marks found on the shirt of the deceased found positive made from sharp cutting weapon (axe) which was recovered from the crime scene. Biological and Serological findings and reports from forensic science laboratory came out positive and are sufficient to point out the culprit.

\section{Results and Discussion}

At the scene of the crime of a murder case, forensic scientists must determine the nature of death (i.e. whether the death is natural or unnatural, and if the death is unnatural, whether it is homicidal, suicidal, or accidental). Extra care has to be taken during 
investigation to rule out any manipulation. The physical evidence collected at the crime scene is used to answer one other important question, how the criminal can be linked with the crime. Many cases have been reported in which the murder is solved instantly at the crime scene. On the basis of our observation and findings; it was concluded that involvement of the deceased brother in the murder was not ruled-out. Our observations provided a new direction to the investigation and investigating officer of the case was guided accordingly and the brother of deceased was interrogated. He had no answer for our findings and confessed that how he executes the murder of his own brother.

\section{Conclusion}

The crime scene is a treasure of clues and proper examination of spot, collection of physical evidence and their evaluation could help to establish the real fact. While dealing with such cases of homicidal death, the forensic expert should adopt a scientific approach to evaluate the incidence with logical and systematic approach at the scene of crime and during examination of the dead body, by using his/her common sense and experience to reconstruct the actual happening. A forensic expert must have the knowledge and skills to understand fully the various causes and manner of death. One can achieve the quality by visiting as many cases (scene of crime) as possible.

\section{Acknowledgement}

Author expresses his deep gratitude to Director, State Forensic Science Laboratory, Sagar (India) for giving me opportunity to prepare this case report. Author would like to thank all Senior Scientific officer from State Forensic Science Laboratory, Sagar and Regional Forensic Science Laboratory, Bhopal for the forensic laboratory examination. Author is very grateful to Shri R.A. Chaubey, IPS, Dr. Himani Khanna IPS, Shri Rajiv Chaturvedi DSP for great supporting hand during investigation finally forensic team members for assisting me at crime scene.

\section{References}

1. Bevel VH, Gardner RM (2008) Bloodstain Pattern Analysis: With an Introduction to Crime Scene Reconstruction. CRC Press, 3rd (Edn).

2. James SH, Eckert WG (1998) Interpretation of Bloodstain Evidence at Crime Scenes, CRC, Boca Raton, Florida, USA.

3. Wolson TL (1995) Documentation of bloodstain pattern evidence. J Forensic Ident 45(4): 396-408.

4. Peschel O, Kunz SN, Rothschild MA, Mutzel E (2011) Blood stain pattern analysis. Forensic Sci Med Pathol 7(3): 257-270.

5. James SH, Kish PE, Sutton TP (2005) Principles of Bloodstain Pattern Analysis: Theory and Practice, CRC Taylor \& Francis, Boca Raton, Florida, USA.

6. Knock C, Davison M (2007) Predicting the position of the source of blood stains for angled impacts. J Forensic Sci 52(5): 1044-1049.

7. Kristina P (2017) Blood as an Important Tool in Criminal Investigation J Forensic Sci \& Criminal Inves 3(2): 01-03.
To Submit Your Article Click Here: Submit Article

DOI: 10.32474/PRJFGS.2018.02.000129

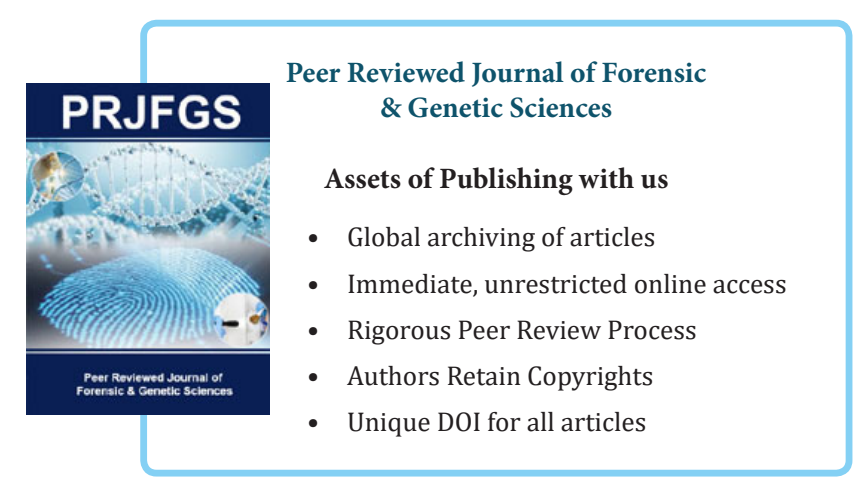

